\title{
构筑苯乙烯轴手性一一高效的醛亚胺临时导向基
}

\author{
冯 佳 顾振华*
}

(中国科学技术大学化学系 合肥微尺度物质科学国家研究中心 合肥 230026)

\section{Construction of Axially Chiral Styrenes_-Highly Efficient Amino Amide Transient Directing Group}

\author{
Feng, Jia Gu, Zhenhua*
}

(Hefei National Laboratory for Physical Sciences at the Microscale, and Department of Chemistry, University of Science and Technology of China, Hefei 230026)

轴手性结构是一些催化剂和配体的优势骨架, 其在 材料化学中的应用也越来越受到重视. 轴手性结构也广 泛地存在于天然产物、生理活性药物之中. 轴手性化合 物不仅表现出不同的生理活性, 有时还展现出对不同受 体的选择性. 早在 1940 年 Adams 和 Miller ${ }^{[1]}$ 就提出了苯 乙烯结构的阻旋手性现象, 1991 年 Fuji 等 ${ }^{[2]}$ 推测了烷基 芳基酮烯醇化后可能形成轴手性苯乙烯中间体，但目前 这类结构的应用远远落后于联芳基轴手性化合物, 其催 化不对称合成也未受到广泛关注, 合成方法也相对有 限.

虽然轴手性结构的稳定性受到取代基的数量、立体 位阻和电性等因素的影响 ${ }^{[3]}$, 但总体而言从芳基-芳基 轴手性到基于烯烃的苯乙烯衍生物的轴手性稳定性是 降低的; 从环状烯烃到开链烯烃, 稳定性会进一步降低, 合成难度更大(图 1). 近年来化学家们对烯基-芳烃轴手 性的催化不对称合成展开了研究. 2016 年 $\mathrm{Gu}$ 课题组 ${ }^{[4]}$ 实现了芳基卤化物与卡宾交叉偶联反应合成基于二氢 萗结构的轴手性分子, 2017 年 Smith 课题组 ${ }^{[5]}$ 通过相转 移催化烷基化实现了二氢萗结构的轴手性分子的高效 构建. 2017 年 Tan 课题组 ${ }^{[6]}$ 通过手性氨基醇衍生物实现 了开链苯乙烯轴手性结构的构建, 之后 Yan 课题组 ${ }^{[7]}$ 通 过炔基萗酚形成联烯轴手性中间体，进而通过加成反应 构建开链苯乙烯轴手性分子.

浙江大学化学系史炳锋课题组 ${ }^{[8]}$ 长期以来一直专注 于过渡金属催化的碳-氢官能化反应研究. 近期他们课 题组通过芳基醛亚胺作为临时导向基, 高效地实现了一 系列轴手性分子的构建. 最近, 他们课题组 ${ }^{[9,10]}$ 将该策

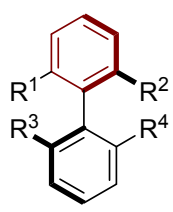

芳基-芳基

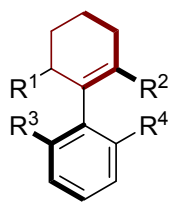

环状烯基-芳基<smiles>[R]/C=C(/[R])c1c([R])cccc1I</smiles>

开链烯基-芳基

趋势: 构象稳定性降低, 轴手性旋转能垒降低

图 1 联芳基、烯基-芳烃轴手性结构

Figure 1 Aryl-aryl and vinyl-arene axially chiral structures 略成功地应用于开链苯乙烯类轴手性结构的构建(Eq. $1)$.

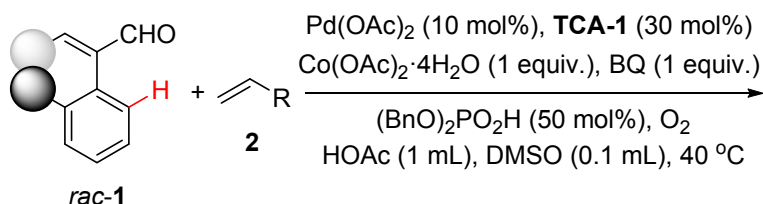

rac-1

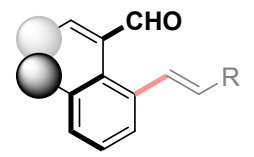

28 examples

up to $95 \%$ yield up to $99 \%$ ee

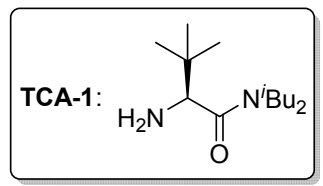

通过 $\mathrm{C}-\mathrm{H}$ 官能团化的策略实现开链苯乙烯轴手性 的构建面临着两大挑战: $\mathrm{C}-\mathrm{H}$ 键活化通常需要的较高 的温度会破坏手性轴的稳定性，不利于反应中轴手性的

* Corresponding author. E-mail: zhgu@ustc.edu.cn. Published online April 26, 2020. 
控制; 底物中丙烯醛含有诸多反应位点, 需要精准调控 反应条件来控制副产物的生成. 经过一系列的条件篮 选, 作者选用消旋的芳基取代的丙烯醛 1 和烯烃化合物 2 作为底物, 在醋酸钯、手性叔丁氨酸衍生的氨基酰胺 和氧气的条件下, 使用醋酸钴和苯醌作为共同氧化剂, 芐氧基膦酸调节体系酸性, 在醋酸和二甲基亚砜的混合 溶剂中, 实现了一系列取代的开链苯乙烯化合物的高效 不对称合成，反应底物适用性好，对映选择性高达 $99 \%$.

作者将合成的亚胺化合物 3 和等物质的量的醋酸钯 反应，得到了环钯中间体 $\mathbf{4}, \mathrm{X}$ 射线单晶衍射表明，其已 经很好地诱导了烯基-芳烃轴手性. 中间体 $\mathbf{4}$ 代替醋酸 钯和手性氨基酰胺, 仍可以较好地诱导对映选择性. 结 合动力学同位素效应实验 $(\mathrm{KIE}=2.3)$, 作者认为 $\mathrm{C}-\mathrm{H}$ 键的断裂是手性控制的关键步骤, 并且是反应的决速步 (Scheme 1).

为了验证合成策略的实用性, 作者实现了轴手性芳 基烯烃产物的克级制备, 并且完成了产物的多官能团转 化(Scheme 2). 作者进一步将衍生化的轴手性烯基羧酸 化合物成功地应用到钴和手性羧酸共催化的不对称的 $\mathrm{C}\left(\mathrm{sp}^{3}\right)-\mathrm{H}$ 胺化反应中, 以较好的收率得到中等对映选 择性 $(82: 18, e r)$ 的产物. 对比联芳基轴手性羧酸催化 剂, 轴手性烯基羧酸催化剂表现出了较好的优势.

综上, 史炳锋课题组发展了一种高效实用的通过钯 催化的 $\mathrm{C}-\mathrm{H}$ 烯基化反应构建开链芳基烯烃轴手性化合

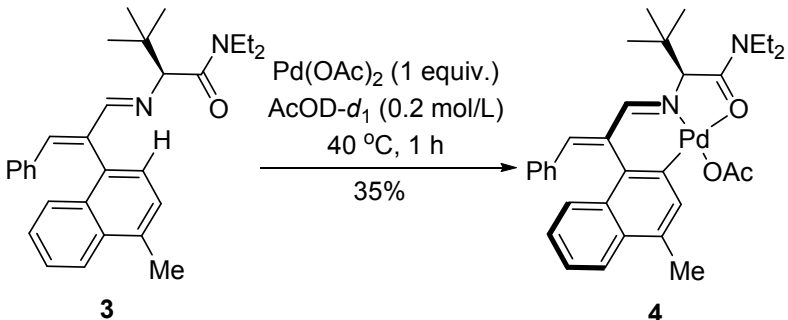

4 (30 mol\%), BQ (1 equiv.)<smiles>CC1=CC=[C+]c2c1cccc2/C(C=O)=C\c1ccccc1</smiles>

图式 1 反应机理研究

Scheme 1 Mechanistic studies

物的新方法. 该方法使用了大位阻的手性醛亚胺临时导 向基团, 为合成在不对称催化中有重要应用的烯基轴手 性化合物提供了一种新思路. 虽然到目前为止基于烯烃 结构的轴手性研究相对较少, 但是我们有理由相信该类 分子在不对称催化以及其它类型手性化合物构建方面 必将有越来越多的应用.

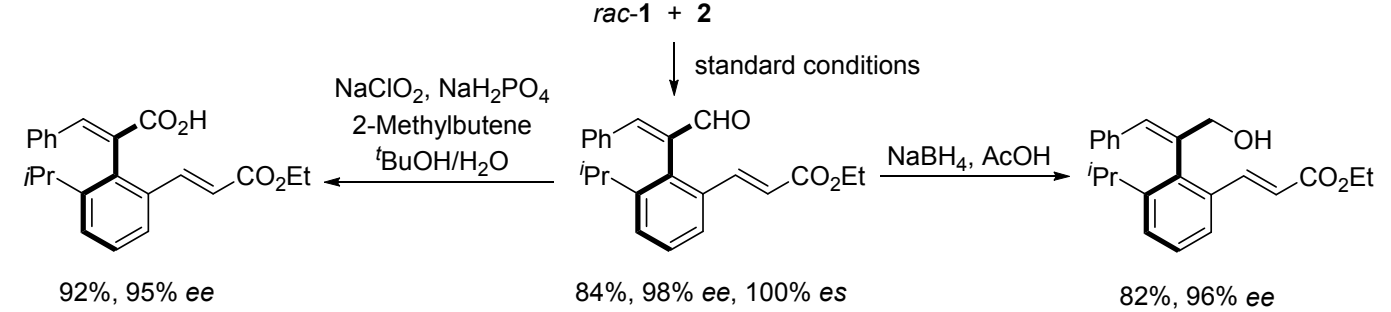

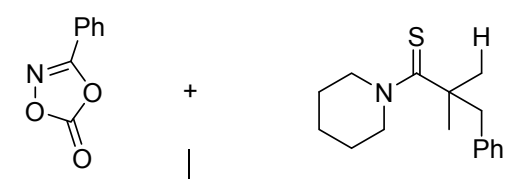

$\left[\mathrm{Cp}{ }^{*} \mathrm{Co}(\mathrm{MeCN})_{3}\right]\left[\mathrm{SbF}_{6}\right]_{2}(5 \mathrm{~mol} \%)$ CCA $(10 \mathrm{~mol} \%)$, oDCB MS $13 \mathrm{X}, 40^{\circ} \mathrm{C}, 24 \mathrm{~h}$<smiles>CC(CNC(=O)c1ccccc1)(C(=S)N1CCCCC1)c1ccccc1</smiles>

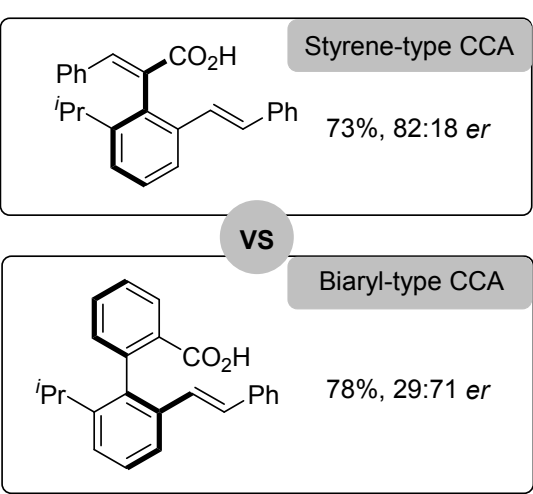

图式 2 苯乙烯类轴手性产物的转化和应用

Scheme 2 Synthetic applications of axially chiral styrenes

\section{References}

[1] Adams, R.; Miller, M. W. J. Am. Chem. Soc. 1940, 62, 53.
[2] Kawabata, T.; Yahiro, K.; Fuji, K. J. Am. Chem. Soc. 1991, 113, 9694.

[3] Bringmann, G.; Mortimer, A. J. P.; Keller, P. A.; Gresser, M. J.; 
Garner, J.; Breuning, M. Angew. Chem., Int. Ed. 2005, 44, 5384.

[4] Feng, J.; Li, B.; He, Y.; Gu, Z. Angew. Chem., Int. Ed. 2016, 55, 2186.

[5] Jolliffe, J. D.; Armstrong, R. J.; Smith, M. D. Nat. Chem. 2017, 9, 558

[6] Zheng, S.-C.; Wu, S.; Zhou, Q.; Chung, L.W.; Ye, L.; Tan, B. Nat. Commun. 2017, 8, 15238.

[7] Jia, S.; Chen, Z.; Zhang, N.; Tan, Y.; Liu, Y.; Deng, J.; Yan, H. J.
Am. Chem. Soc. 2018, 140, 7056.

[8] Yao, Q.-J.; Zhang, S.; Zhan, B.-B.; Shi, B.-F. Angew. Chem., Int. Ed. 2017, 56, 6617.

[9] Song, H.; Li, Y.; Yao, Q.-J.; Jin, L.; Liu, L.; Liu, Y.-H.; Shi, B.-F. Angew. Chem., Int. Ed. 2020, 59, 6576.

[10] Jin, L.; Yao, Q.-J.; Xie, P.-P.; Li, Y.; Zhan, B.-B.; Han, Y.-Q.; Hong, X.; Shi, B.-F. Chem 2020, 6, 497.

(Cheng, F.) 